\title{
An approximate subgradient algorithm for unconstrained nonsmooth, nonconvex optimization
}

\author{
Adil Bagirov • Asef Nazari Ganjehlou
}

Received: date / Accepted: date

\begin{abstract}
In this paper a new algorithm for minimizing locally Lipschitz functions is developed. Descent directions in this algorithm are computed by solving a system of linear inequalities. The convergence of the algorithm is proved for quasidifferentiable semismooth functions. We present the results of numerical experiments with both regular and nonregular objective functions. We also compare the proposed algorithm with two different versions of the subgradient method using the results of numerical experiments. These results demonstrate the superiority of the proposed algorithm over the subgradient method.
\end{abstract}

Keywords nonsmooth optimization, subdifferential, semismooth functions, quasidifferentiable functions.

Mathematics Subject Classification (2000) 65K05, 90C25.

\section{Introduction}

Consider the following unconstrained minimization problem:

$$
\text { minimize } f(x) \text { subject to } x \in \mathbb{R}^{n}
$$

where the objective function $f$ is locally Lipschitz. Different algorithms have been proposed to solve Problem (1). Bundle-type methods [14,16,18,19,21,23,27,28], a gra-

\section{Adil Bagirov}

Centre for Informatics and Applied Optimization, School of Information Technology and Mathematical Sciences, University of Ballarat, University Drive, Mount Helen, PO Box 663, Ballarat, Victoria, 3353, Australia

Tel.: +61-3-53279330

Fax: +61-3-53279289

E-mail: a.bagirov@ballarat.edu.au

Asef Nazari Ganjehlou

Centre for Informatics and Applied Optimization, School of Information Technology and Mathematical Sciences, University of Ballarat, University Drive, Mount Helen, PO Box 663, Ballarat, Victoria, 3353, Australia

E-mail: a.nazari@ballarat.edu.au 
dient sampling algorithm [11], algorithms based on smoothing techniques [24] and the discrete gradient method [3-7] are among them.

The subgradient method is a very simple algorithm for minimizing a nonsmooth convex function (see, [10] and [26] for details). This method uses step-lengths that are fixed ahead of time and it does not contain a line search procedure. The subgradient method is not a descent method. For some problems it is extremely inefficient. However, it is simple and can be applied to a far wider variety problems. Therefore it is important to develop minimization algorithms based on the subgradient methods which are still quite simple, easy to implement and on the same time are more efficient than the subgradient algorithms and applicable to a wider class of minimization problems.

In this paper we present one such algorithm. This algorithm can be applied for minimizing nonconvex, nonsmooth functions. In this algorithm descent directions are computed by solving a system of linear inequalities. The latter problem is solved using the subgradient method. Armijo-type line search technique is used to find step-lengths. The convergence of the proposed algorithm is proved for quasidifferentiable semismooth functions. We also present the results of numerical experiments.

The structure of the paper is as follows. Section 2 provides some necessary preliminaries. We describe an approach to approximate subgradients in Section 3 and an algorithm for the computation of descent directions in Section 4. An approximate subgradient algorithm is discussed in Section 5. We present the results of numerical experiments and their discussion in Section 6. Section 7 concludes the paper.

\section{Preliminaries}

\subsection{The Clarke subdifferential}

Let $f$ be a locally Lipschitz function defined on $\mathbb{R}^{n}$. Clarke introduced the notion of subdifferential for such functions (see, for example, [12]). Since these functions are differentiable almost everywhere, we can define for them a Clarke subdifferential as follows:

$$
\partial f(x)=\operatorname{co}\left\{v \in \mathbb{R}^{n}: \exists\left(x^{k} \in D(f), x^{k} \rightarrow x, k \rightarrow+\infty\right): v=\lim _{k \rightarrow+\infty} \nabla f\left(x^{k}\right)\right\},
$$

here $D(f)$ denotes the set where $f$ is differentiable, co denotes the convex hull of a set. It is shown in [12] that the mapping $x \mapsto \partial f(x)$ is upper semicontinuous and bounded on bounded sets.

The generalized directional derivative of $f$ at $x$ in the direction $g$ is defined as

$$
f^{0}(x, g)=\limsup _{y \rightarrow x, \alpha \rightarrow+0} \alpha^{-1}[f(y+\alpha g)-f(y)] .
$$

If the function $f$ is locally Lipschitz, then the generalized directional derivative exists and

$$
f^{0}(x, g)=\max \{\langle v, g\rangle: v \in \partial f(x)\} .
$$

A function $f$ is called a regular function on $\mathbb{R}^{n}$, if it is differentiable with respect to any direction $g \in \mathbb{R}^{n}$ and $f^{\prime}(x, g)=f^{0}(x, g)$ for all $x, g \in \mathbb{R}^{n}$ where $f^{\prime}(x, g)$ is a derivative of the function $f$ at the point $x$ in the direction $g$ :

$$
f^{\prime}(x, g)=\lim _{\alpha \rightarrow+0} \alpha^{-1}[f(x+\alpha g)-f(x)] .
$$


For a point $x$ to be a minimum point of the function $f$ on $\mathbb{R}^{n}$, it is necessary that $0 \in \partial f(x)$.

\subsection{Semismooth functions}

A function $f: \mathbb{R}^{n} \rightarrow \mathbb{R}^{1}$ is called semismooth at $x \in \mathbb{R}^{n}$, if it is locally Lipschitz at $x$ and for every $g \in \mathbb{R}^{n}$, the limit

$$
\lim _{v \in \partial f\left(x+\alpha g^{\prime}\right), g^{\prime} \rightarrow g, \alpha \rightarrow+0}\langle v, g\rangle
$$

exists. The class of semismooth functions contains convex, concave, max-type and mintype functions [22]. The semismooth function $f$ is directionally differentiable and

$$
f^{\prime}(x, g)=\lim _{v \in \partial f\left(x+\alpha g^{\prime}\right), g^{\prime} \rightarrow g, \alpha \rightarrow+0}\langle v, g\rangle .
$$

2.3 Quasidifferentiable functions

A function $f$ is called quasidifferentiable at a point $x$ if it is locally Lipschitz, directionally differentiable at this point and there exist convex, compact sets $\underline{\partial} f(x)$ and $\bar{\partial} f(x)$ such that:

$$
f^{\prime}(x, g)=\max _{u \in \underline{\partial} f(x)}\langle u, g\rangle+\min _{v \in \bar{\partial} f(x)}\langle v, g\rangle .
$$

The set $\underline{\partial} f(x)$ is called a subdifferential, the set $\bar{\partial} f(x)$ is called a superdifferential and the pair $[\underline{\partial} f(x), \bar{\partial} f(x)]$ is called a quasidifferential of the function $f$ at a point $x$ [13].

\section{Approximation of subgradients}

In this section we consider an approach to approximate subdifferentials. This approach is based on the notion of a discrete gradient, which was introduced in [1] (see also [2, $3,5]$ ). Here all propositions are given without proofs (for the proofs see [6] and [7]).

In this and subsequent sections we will use the following notations:

$$
\begin{gathered}
S_{\varepsilon}(x)=\left\{y \in \mathbb{R}^{n}:\|x-y\|<\varepsilon\right\}, \quad \bar{S}_{\varepsilon}(x)=\left\{y \in \mathbb{R}^{n}:\|x-y\| \leq \varepsilon\right\} \\
S_{\varepsilon}=S_{\varepsilon}(0), \quad \bar{S}_{\varepsilon}=\bar{S}_{\varepsilon}(0), \quad S_{1}=\left\{g \in \mathbb{R}^{n}:\|g\|=1\right\}, \\
\mathbb{R}^{+}=\left\{t \in \mathbb{R}^{1}: t>0\right\} .
\end{gathered}
$$

Let $G=\left\{e \in \mathbb{R}^{n}: e=\left(e_{1}, \ldots, e_{n}\right),\left|e_{j}\right|=1, j=1, \ldots, n\right\}$ be a set of all vertices of the unit hypercube in $\mathbb{R}^{n}$. We take $e \in G$ and consider the sequence of $n$ vectors $e^{j}=e^{j}(\alpha), j=1, \ldots, n$ with $\alpha \in(0,1]$ :

$$
\begin{aligned}
e^{1} & =\left(\alpha e_{1}, 0, \ldots, 0\right), \\
e^{2} & =\left(\alpha e_{1}, \alpha^{2} e_{2}, 0, \ldots, 0\right), \\
\ldots & =\ldots \ldots \ldots \\
e^{n} & =\left(\alpha e_{1}, \alpha^{2} e_{2}, \ldots, \alpha^{n} e_{n}\right) .
\end{aligned}
$$


Assume $\lambda>0$ be a given number. Let

$$
P=\left\{z: z: \mathbb{R}^{+} \rightarrow \mathbb{R}^{+}, \beta^{-1} z(\beta) \rightarrow+0, \beta \rightarrow+0\right\}
$$

be the set of all univariate positive infinitesimal functions. We take any $g \in S_{1}, e \in G$ and compute $i \in\{1, \ldots, n\}$ such that $\left|g_{i}\right|=\max \left\{\left|g_{k}\right|, \quad k=1, \ldots, n\right\}$. For given $x \in \mathbb{R}^{n}$ and $z \in P$ consider a sequence of $n+1$ points:

$$
\begin{aligned}
& x^{0}=x+\lambda g, \\
& x^{1}=x^{0}+z(\lambda) e^{1}(\alpha), \\
& \ldots=\ldots \\
& x^{n}=x^{0}+z(\lambda) e^{n}(\alpha) .
\end{aligned}
$$

Let $f$ be a function defined on $\mathbb{R}^{n}$.

Definition 1 The discrete gradient of the function $f$ at the point $x \in \mathbb{R}^{n}$ is the vector $\Gamma^{i}(x, g, e, z, \lambda, \alpha)=\left(\Gamma_{1}^{i}, \ldots, \Gamma_{n}^{i}\right) \in \mathbb{R}^{n}, g \in S_{1}$ with the following coordinates:

$$
\begin{gathered}
\left.\Gamma_{j}^{i}=\left[z(\lambda) \alpha^{j} e_{j}\right)\right]^{-1}\left[f\left(x^{j}\right)-f\left(x^{j-1}\right)\right], \quad j=1, \ldots, n, \quad j \neq i, \\
\Gamma_{i}^{i}=\left(\lambda g_{i}\right)^{-1}\left[f(x+\lambda g)-f(x)-\lambda \sum_{j=1, j \neq i}^{n} \Gamma_{j}^{i} g_{j}\right] .
\end{gathered}
$$

It follows from Definition 1 that

$$
f(x+\lambda g)-f(x)=\lambda\left\langle\Gamma^{i}(x, g, e, z, \lambda, \alpha), g\right\rangle
$$

for all $g \in S_{1}, e \in G, z \in P, \lambda>0, \alpha>0$.

Remark 1 One can see that the discrete gradient is defined with respect to a given direction $g \in S_{1}$ and in order to compute it, first we define a sequence of points $x^{0}, \ldots, x^{n}$ and compute the values of the function $f$ at these points that is we compute $n+2$ values of this function including the point $x$. The $i$-th coordinate is defined so that to satisfy the equality (2) which can be considered as some version of the mean value theorem.

Proposition 1 Let $f$ be a locally Lipschitz function defined on $\mathbb{R}^{n}$ and $L>0$ is its Lipschitz constant. Then for any $x \in \mathbb{R}^{n}, g \in S_{1}, e \in G, \lambda>0, z \in P, \alpha>0$

$$
\left\|\Gamma^{i}\right\| \leq \bar{C}, \quad \bar{C}=C(n) L, \quad C(n)=\left(n^{2}+2 n^{3 / 2}-2 n^{1 / 2}\right)^{1 / 2} .
$$

For a given $\alpha>0$ we define the following set:

$$
\begin{gathered}
B(x, \alpha)=\left\{v \in \mathbb{R}^{n}: \exists\left(g \in S_{1}, e \in G, z_{k} \in P, z_{k} \rightarrow+0, \lambda_{k} \rightarrow+0, k \rightarrow+\infty\right),\right. \\
\left.v=\lim _{k \rightarrow+\infty} \Gamma^{i}\left(x, g, e, z_{k}, \lambda_{k}, \alpha\right)\right\} .
\end{gathered}
$$

From now on we consider a function $f$ defined on $\mathbb{R}^{n}$ and assume that this function is quasidifferentiable. We also assume that both sets $\underline{\partial} f(x)$ and $\bar{\partial} f(x)$ are polytopes at any $x \in \mathbb{R}^{n}$ that is at a point $x \in \mathbb{R}^{n}$ there exist sets

$$
A=\left\{a^{1}, \ldots, a^{m}\right\}, \quad a^{i} \in \mathbb{R}^{n}, \quad i=1, \ldots, m, m \geq 1,
$$




$$
B=\left\{b^{1}, \ldots, b^{p}\right\}, \quad b^{j} \in \mathbb{R}^{n}, \quad j=1, \ldots, p, p \geq 1
$$

such that

$$
\underline{\partial} f(x)=\operatorname{co} A, \quad \bar{\partial} f(x)=\operatorname{co} B .
$$

We denote by $\mathcal{F}$ the class of all semismooth, quasidifferentiable functions whose subdifferential and superdifferential are polytopes at any $x \in \mathbb{R}^{n}$. This class contains, for example, functions represented as a maximum, minimum or max-min of a finite number of smooth functions.

Proposition 2 Assume that $f \in \mathcal{F}$. Then at a given point $x$ there exists $\alpha_{0}>0$ such that

$$
\operatorname{coB}(x, \alpha) \subset \partial f(x), \forall \alpha \in\left(0, \alpha_{0}\right]
$$

Remark 2 After fixing $g \in S_{1}$ and $e \in G$ the discrete gradient contains three parameters: $\lambda>0, z \in P$ and $\alpha>0$. The function $z \in P$ is used to exploit semismoothness of the function $f$ and it can be chosen sufficiently small. If $f \in \mathcal{F}$, then for any $\delta>0$ there exists $\alpha_{0}>0$ such that $\alpha \in\left(0, \alpha_{0}\right]$ for all $y \in S_{\delta}(x)$. In the sequel we assume that $z \in P$ and $\alpha>0$ are sufficiently small.

For a given $\lambda>0$ consider the following set at a point $x \in \mathbb{R}^{n}$ :

$$
D_{0}(x, \lambda)=\operatorname{clco}\left\{v \in \mathbb{R}^{n}: \exists\left(g \in S_{1}, e \in G, z \in P\right): \quad v=\Gamma^{i}(x, g, e, \lambda, z, \alpha)\right\} .
$$

Proposition 1 implies that for a locally Lipschitz function, the set $D_{0}(x, \lambda)$ is compact and convex for any $x \in \mathbb{R}^{n}$.

Corollary 1 Assume that $f \in \mathcal{F}$ and in the equality

$$
f(x+\lambda g)-f(x)=\lambda f^{\prime}(x, g)+o(\lambda, g), g \in S_{1},
$$

$\lambda^{-1} o(\lambda, g) \rightarrow 0$ as $\lambda \rightarrow+0$ uniformly with respect to $g \in S_{1}$. Then for any $\varepsilon>0$ there exists $\lambda_{0}>0$ such that $D_{0}(x, \lambda) \subset \partial f(x)+S_{\varepsilon}$ for all $\lambda \in\left(0, \lambda_{0}\right)$.

Corollary 1 shows that the set $D_{0}(x, \lambda)$ is an approximation to the subdifferential $\partial f(x)$ for sufficiently small $\lambda>0$. However, it is true at a given point $x$ but not in some its neighborhood. In order to get convergence results for a minimization algorithm based on discrete gradients we need some relationship between the sets $D_{0}(x, \lambda)$ and $\partial f(x)$ in some neighborhood of a given point $x$. We will consider functions satisfying the following assumption.

Assumption 1 Let $x \in \mathbb{R}^{n}$ be a given point. For any $\varepsilon>0$ there exist $\delta>0$ and $\lambda_{0}>0$ such that

$$
D_{0}(y, \lambda) \subset \partial f\left(x+\bar{S}_{\varepsilon}\right)+S_{\varepsilon}
$$

for all $y \in S_{\delta}(x)$ and $\lambda \in\left(0, \lambda_{0}\right)$. Here

$$
\partial f\left(x+\bar{S}_{\varepsilon}\right)=\bigcup_{y \in \bar{S}_{\varepsilon}(x)} \partial f(y) .
$$

Remark 3 The set $D_{0}(x, \lambda), \lambda>0$ can be used to compute descent directions of the function $f$. However, the computation of this set is very time-consuming. In the next section we propose an algorithm for computation of descent directions which uses only a few discrete gradients from $D_{0}(x, \lambda)$. 


\section{Computation of descent directions}

In this section we propose an algorithm for the computation of descent directions.

Let $z \in P, \lambda>0, \alpha \in(0,1]$, numbers $c \in(0,1)$ and $\delta>0$ be given.

Algorithm 1 An algorithm for the computation of the descent direction.

Step 1. Choose any $g^{1} \in S_{1}, e \in G$, compute $i=\operatorname{argmax}\left\{\left|g_{j}\right|, j=1, \ldots, n\right\}$ and a discrete gradient $v^{1}=\Gamma^{i}\left(x, g^{1}, e, z, \lambda, \alpha\right)$. Set $\tilde{D}_{1}(x)=\left\{v^{1}\right\}$ and $k=1$.

Step 2. Compute the direction $g \in \mathbb{R}^{n}$ as a solution to the following system:

$$
\left\langle v^{i}, g\right\rangle+\delta \leq 0, i=1, \ldots, k, \quad g \in S_{1}
$$

Step 3. If the system (5) is not solvable, then stop. Otherwise compute $\bar{g}$ as a solution to this system and go to Step 4.

Step 4. If

$$
f(x+\lambda \bar{g})-f(x) \leq-c \delta \lambda,
$$

then stop. Otherwise set $g^{k+1}=\bar{g}$ and go to Step 5 .

Step 5. Compute $i=\operatorname{argmax}\left\{\left|g_{j}^{k+1}\right|: j=1, \ldots, n\right\}$ and a discrete gradient

$$
v^{k+1}=\Gamma^{i}\left(x, g^{k+1}, e, z, \lambda, \alpha\right),
$$

construct the set $\tilde{D}_{k+1}(x)=\operatorname{co}\left\{\tilde{D}_{k}(x) \bigcup\left\{v^{k+1}\right\}\right\}$, set $k=k+1$ and go to Step 2 .

Some explanation to Algorithm 1 is necessary. In Step 1 we compute the discrete gradient with respect to an initial direction $g^{1} \in S_{1}$. In Step 2 we find a solution to the system of linear inequalities (5) with additional condition $g \in S_{1}$ (below we will discuss algorithms for solving the system (5)). If the system is not solvable, then in Step 3 we accept the point $x \in \mathbb{R}^{n}$ as an approximate stationary point and the algorithm stops (see Remark 4, below). If the system is solvable, then we compute a new search direction $\bar{g}$ and in Step 4 we check whether this direction is a descent direction. If it is the algorithm stops and the descent direction has been computed, otherwise we compute another discrete gradient with respect to this direction in Step 5 and update the set $\tilde{D}_{k}(x)$. At each iteration $k$ we improve the approximation of the subdifferential of the function $f$.

We will show that Algorithm 1 is terminating that is after finite number of steps either we find that the point $x$ is an approximate stationary point or we compute the descent direction. First, we will prove the following propositions.

Proposition 3 If the system (5) is not solvable, then

$$
\min _{v \in \tilde{D}_{k}(x)}\|v\|<\delta
$$


Proof: Let $\tilde{v}$ be a solution to the following problem:

$$
\min \frac{1}{2}\|v\|^{2} \quad \text { subject to } v \in \tilde{D}_{k}(x)
$$

If $\tilde{v}=0$ then the proof is straightforward. So we assume that $\tilde{v} \neq 0$. Then it follows from the necessary condition for a minimum that

$$
\langle\tilde{v}, v-\tilde{v}\rangle \geq 0, \quad \forall v \in \tilde{D}_{k}(x)
$$

which means

$$
\|\tilde{v}\|^{2} \leq\langle\tilde{v}, v\rangle, \quad \forall v \in \tilde{D}_{k}(x)
$$

Since the system (5) is not solvable we get

$$
\max _{i=1, \ldots, k}\left\langle v^{i}, g\right\rangle>-\delta, \quad \forall g \in S_{1}
$$

Consider $g=-\frac{\tilde{v}}{\|\tilde{v}\|}$. Then there exists $i \in\{1, \ldots, k\}$ such that

$$
\left\langle\tilde{v}, v^{i}\right\rangle<\delta\|\tilde{v}\|
$$

Then the proof follows from (8).

Remark 4 It follows from Proposition 3 that if in Step 2 of Algorithm 1 the system (5) is not solvable, then the point $x \in \mathbb{R}^{n}$ can be considered as an approximate solution.

Proposition 4 If (7) is satisfied then the system (5) is not solvable.

Proof: Assume the contrary that is (7) holds, but the system (5) has a solution. The latter means that there exists $g \in S_{1}$ such that

$$
\left\langle v^{i}, g\right\rangle+\delta \leq 0, i=1, \ldots, k .
$$

Let

$$
\|\tilde{v}\|=\min _{v \in \tilde{D}_{k}(x)}\|v\| .
$$

Since $\tilde{v} \in \tilde{D}_{k}(x)$

$$
\tilde{v}=\sum_{i \in I} \alpha_{i} v^{i}, \quad \sum_{i \in I} \alpha_{i}=1, \quad \alpha_{i} \in(0,1], i \in I \subset\{1, \ldots, k\} .
$$

We get

$$
\langle\tilde{v}, g\rangle \leq-\delta
$$

On the other hand

$$
|\langle\tilde{v}, g\rangle| \leq\|\tilde{v}\|\|g\|=\|\tilde{v}\|<\delta
$$

which contradicts (9).

Proposition 5 Let $f$ be a locally Lipschitz function defined on $\mathbb{R}^{n}$. Then in Algorithm 1 one of the stopping criteria will be satisfied after finite number of steps. 
Proof: If both conditions for the termination of the algorithm are not satisfied, then a new discrete gradient $v^{k+1} \notin \tilde{D}_{k}(x)$. Indeed, in this case

$$
f\left(x+\lambda g^{k+1}\right)-f(x)>-c \delta \lambda .
$$

It follows from (2) that

$$
\begin{aligned}
f\left(x+\lambda g^{k+1}\right)-f(x) & =\lambda\left\langle\Gamma^{i}\left(x, g^{k+1}, e, z, \lambda, \alpha\right), g^{k+1}\right\rangle \\
& =\lambda\left\langle v^{k+1}, g^{k+1}\right\rangle
\end{aligned}
$$

and therefore

$$
\left\langle v^{k+1}, g^{k+1}\right\rangle>-c \delta .
$$

Assume that $v^{k+1} \in \tilde{D}_{k}(x)$. Since $g^{k+1} \in S_{1}$ is a solution to the system (5)

$$
\left\langle v^{i}, g^{k+1}\right\rangle+\delta \leq 0, i=1, \ldots, k
$$

we have

$$
\left\langle v^{k+1}, g^{k+1}\right\rangle \leq-\delta
$$

which contradicts (10). The latter means that $v^{k+1} \notin \tilde{D}_{k}(x)$.

Now we will show that Algorithm 1 is terminating. Assume the contrary. Then Algorithm 1 generates an infinite sequence $\left\{g^{k}\right\}$ of directions $g^{k} \in S_{1}$. It follows from (10) that

$$
\left\langle v^{k}, g^{k}\right\rangle>-c \delta, \quad \forall k=2,3, \ldots
$$

The latter means that for any $k \in\{2,3, \ldots\}$ the direction $g^{k}$ does not satisfy the system:

$$
\left\langle v^{t}, g\right\rangle+\delta \leq 0, t=1, \ldots, i, i \geq k .
$$

It follows from Proposition 1 that $\|v\| \leq \bar{C}$ for all $v \in \tilde{D}_{k}(x)$. The direction $g^{k+1}$ is a solution to the system

$$
\left\langle v^{i}, g\right\rangle+\delta \leq 0, i=1, \ldots, k .
$$

However, directions $g^{j}, j=2, \ldots, k$ are not solutions to this system. Then we get

$$
\left\|g^{k+1}-g^{j}\right\|>\frac{(1-c) \delta}{\bar{C}}, \forall j=2, \ldots, k .
$$

Indeed, if there exists $j \in\{2, \ldots, k\}$ such that

$$
\left\|g^{k+1}-g^{j}\right\| \leq \frac{(1-c) \delta}{\bar{C}}
$$

then we have

$$
\left|\left\langle v^{j}, g^{k+1}\right\rangle-\left\langle v^{j}, g^{j}\right\rangle\right| \leq(1-c) \delta .
$$

The latter means that

$$
\left\langle v^{j}, g^{j}\right\rangle \leq\left\langle v^{j}, g^{k+1}\right\rangle+(1-c) \delta \leq-c \delta
$$

which contradicts (11). The inequality (12) can be rewritten as follows:

$$
\min _{j=2, \ldots, k}\left\|g^{k+1}-g^{j}\right\|>\frac{(1-c) \delta}{\bar{C}} .
$$

Thus Algorithm 1 generates a sequence $\left\{g^{k}\right\}$ of directions $g^{k} \in S_{1}$ such that the distance between $g^{k}$ and the set of all previous directions is bounded below. Since the set $S_{1}$ is compact, the number of such directions is finite. 
4.1 Solving the system (5)

Step 2 is an important step in Algorithm 1, where we solve the system (5) to find search directions. Different methods have been developed to solve a system of linear inequalities (see, for example, $[15,17]$ ). However, these methods cannot be applied directly to solve the system (5) because of the presence of the additional condition $g \in S_{1}$. In this paper we use the nonsmooth optimization approach. To solve the system (5) we reformulate it as the following optimization problem:

$$
\operatorname{minimize} \varphi_{k}(g)=\max \left\{0,\left\langle v^{j}, g\right\rangle+\delta, j=1, \ldots, k\right\}
$$

subject to

$$
g \in B_{1}=\left\{y \in \mathbb{R}^{n}:\|y\|^{2} \leq 1\right\} .
$$

It is clear that if the system (5) is solvable then there exists $g \in S_{1}$ such that $\varphi_{k}(g)=0$. If it is not solvable then $\varphi_{k}(g)>0$ for all $g \in S_{1}$.

The function $\varphi_{k}$ is convex and piecewise linear and the problem (13)-(14) is convex programming problem. The problem of minimization of the function $\varphi_{k}$ without the constraint (14) can be easily reduced to a linear programming problem. However, linear programming techniques cannot be applied directly to solve the problem (13)-(14) because of the nonlinear constraint (14).

The nonsmooth optimization approach has some advantages. First of all, since the discrete gradients are computed step by step we get the sequence of minimization problems of convex piecewise linear functions over the unit ball. The functions $\varphi_{k}$ are built step by step and one can use the solution in step $j, j<k$ as a starting point in step $(j+1)$ which allows one to reduce the computational effort. We use the subgradient method to solve Problem (13)-(14). Since the minimum value or its lower bound is 0 we can use the following version of the subgradient method [25]:

$$
g^{l+1}=\operatorname{Proj}_{B_{1}}\left(g^{l}-\frac{\varphi_{k}\left(g^{l}\right)}{\left\|w^{l}\right\|^{2}} w^{l}\right),
$$

where $\operatorname{Proj}_{B_{1}}(\cdot)$ is a projection operator onto the set $B_{1}, w^{l} \in \partial \varphi_{k}\left(g^{l}\right)$ is a subgradient of the function $\varphi_{k}$ at the point $g^{l}$. The subgradient $w^{l}$ is computed as follows. First, we compute

$$
R\left(g^{l}\right)=\left\{i \in\{1, \ldots, k\}:\left\langle v^{i}, g^{l}\right\rangle+\delta=\varphi_{k}\left(g^{l}\right)\right\} .
$$

Then the subdifferential of the function $\varphi_{k}$ at the point $g^{l}$ is:

$$
\partial \varphi_{k}\left(g^{l}\right)=\operatorname{co}\left\{v^{i}: i \in R\left(g^{l}\right)\right\} .
$$

Let $l_{0}=\left|R\left(g^{l}\right)\right|$ be the cardinality of the set $R\left(g^{l}\right)$. Then

$$
w^{l}=\frac{1}{l_{0}} \sum_{i \in R\left(g^{l}\right)} v^{i} .
$$

The convergence results for this version of the subgradient method can be found, for example, in [25]. 


\section{The approximate subgradient method}

In this section we describe the approximate subgradient method. Let sequences $\delta_{k}>$ $0, z_{k} \in P, \lambda_{k}>0, \delta_{k} \rightarrow+0, z_{k} \rightarrow+0, \lambda_{k} \rightarrow+0, k \rightarrow+\infty$, sufficiently small number $\alpha>0$ and numbers $c_{1} \in(0,1), c_{2} \in\left(0, c_{1}\right]$ be given.

Algorithm 2 The approximate subgradient method

Step 1. Choose any starting point $x^{0} \in \mathbb{R}^{n}$ and set $k=0$.

Step 2. Set $s=0$ and $x^{k_{s}}=x^{k}$.

Step 3. Apply Algorithm 1 for computation of the descent direction at $x=x^{k_{s}}, \delta=$ $\delta_{k}, z=z_{k}, \lambda=\lambda_{k}, c=c_{1}$. This algorithm terminates after a finite number of iterations $l>0$. As a result we get the system:

$$
\left\langle v^{i}, g\right\rangle+\delta_{k} \leq 0, \quad i=1, \ldots, l, \quad g \in S_{1} .
$$

Step 4. If this system is not solvable put $x^{k+1}=x^{k_{s}}, k=k+1$ and go to Step 2 Otherwise we get the direction $g^{k_{s}} \in S_{1}$ which is a solution to this system and

$$
f\left(x^{k_{s}}+\lambda_{k} g^{k_{s}}\right)-f\left(x^{k_{s}}\right) \leq-c_{1} \lambda_{k} \delta_{k}
$$

Step 5. Construct the following iteration $x^{k_{s+1}}=x^{k_{s}}+\sigma_{s} g^{k_{s}}$, where $\sigma_{s}$ is defined as follows

$$
\sigma_{s}=\operatorname{argmax}\left\{\sigma \geq 0: f\left(x^{k_{s}}+\sigma g^{k_{s}}\right)-f\left(x^{k_{s}}\right) \leq-c_{2} \sigma \delta_{k}\right\} .
$$

Step 6. Set $s=s+1$ and go to Step 3 .

Remark 5 One can see that Algorithm 2 consists of two loops: inner and outer loops. The inner loop consists of Steps 3, 4, 5, 6 and parameters $\delta_{k}, z_{k}, \lambda_{k}$ are fixed in this loop. The outer loop consists of Steps 2, 3 and 5 and the parameters $\delta_{k}, z_{k}, \lambda_{k}$ are updated in this loop. The algorithm each time returns to the outer loop if further improvement of the solution is not possible with the same values of the parameters and they have to be updated to improve the approximation of subgradients.

Remark 6 Unlike the subgradient method the proposed algorithm may use more than one approximate subgradient at each iteration. This makes it similar to bundle-type methods. But on the same time it does not use polyhedral underestimators of the objective function which makes it different from them. Moreover, in this method the subgradient method is applied to find descent directions.

For the point $x^{0} \in \mathbb{R}^{n}$ we consider the set $M\left(x^{0}\right)=\left\{x \in \mathbb{R}^{n}: f(x) \leq f\left(x^{0}\right)\right\}$.

Theorem 1 Assume that $f \in \mathcal{F}$, Assumption 1 is satisfied and the set $M\left(x^{0}\right)$ is bounded for starting points $x^{0} \in \mathbb{R}^{n}$. Then every accumulation point of $\left\{x^{k}\right\}$ belongs to the set $X^{0}=\left\{x \in \mathbb{R}^{n}: 0 \in \partial f(x)\right\}$. 
Proof: Since the function $f$ is locally Lipschitz and the set $M\left(x^{0}\right)$ is bounded

$$
f_{*}=\inf \left\{f(x): x \in \mathbb{R}^{n}\right\}>-\infty .
$$

First we show that the inner loop stops after finite number of steps. In other words for any $k>0$ there exists $s=s_{k} \geq 0$ such that the system (15) becomes unsolvable at $x^{k_{s}}$. Assume the contrary that is there exists $k>0$ such that the inner loop is infinite for this $k$. This implies that the system (15) is solvable and the inequality (16) is satisfied for all $s \geq 0$. Since $c_{2} \in\left(0, c_{1}\right]$ it follows from (16) that $\sigma_{s} \geq \lambda_{k}$. Then we can write

$$
\begin{aligned}
f\left(x^{k_{s+1}}\right)-f\left(x^{k_{s}}\right) & \leq-c_{2} \sigma_{s} \delta_{k} \\
& <-c_{2} \sigma_{s}\left\|v^{k_{s}}\right\| \\
& \leq-c_{2} \lambda_{k}\left\|v^{k_{s}}\right\| .
\end{aligned}
$$

If the system (15) is solvable for any $s$ then it follows from Propositions 3 and 4 that $\left\|v^{k_{s}}\right\| \geq \delta_{k}$ and

$$
f\left(x^{k_{s+1}}\right)-f\left(x^{k_{s}}\right) \leq-c_{2} \lambda_{k} \delta_{k}
$$

or

$$
f\left(x^{k_{s+1}}\right) \leq f\left(x^{k_{0}}\right)-(s+1) c_{2} \lambda_{k} \delta_{k} .
$$

Since $\lambda_{k}>0$ and $\delta_{k}>0$ are fixed for any $k>0$ it follows from (18) that $f\left(x^{k_{s}}\right) \rightarrow-\infty$ as $s \rightarrow+\infty$. This contradicts (17), that is the inner loop stops after a finite number of steps. This implies that for any $k>0$ there exists $s=s_{k} \geq 0$ such that the system (15) is not solvable at $x^{k_{s}}$. It follows from Proposition 3 that

$$
\left\|v^{k_{s}}\right\|=\min _{v \in \tilde{D}^{k_{s}}\left(x^{k_{s}}\right)}\|v\|<\delta_{k}
$$

At the end of $k$-th inner loop we get a point $x^{k+1}=x^{k_{s}}$ and

$$
\min _{v \in \tilde{D}_{l}\left(x^{k+1}\right)}\|v\|<\delta_{k}
$$

Since $\tilde{D}_{l}\left(x^{k+1}\right) \subset D_{0}\left(x^{k+1}, \lambda_{k}\right)$,

$$
\min _{v \in D_{0}\left(x^{k+1}, \lambda_{k}\right)}\|v\|<\delta_{k}
$$

Replacing $k+1$ by $k$ we get

$$
\min _{v \in D_{0}\left(x^{k}, \lambda_{k-1}\right)}\|v\|<\delta_{k-1}
$$

Since $\left\{f\left(x^{k}\right)\right\}$ is a decreasing sequence, $x^{k} \in M\left(x^{0}\right)$ for all $k>0$. Then the sequence $\left\{x^{k}\right\}$ is bounded and therefore it has at least one accumulation point. Assume $x^{*}$ is any accumulation point of the sequence $\left\{x^{k}\right\}$ and $x^{k_{i}} \rightarrow x^{*}$ as $i \rightarrow+\infty$. Then we have from (19)

$$
\min _{v \in D_{0}\left(x^{\left.k_{i}, \lambda_{k_{i}-1}\right)}\right.}\|v\| \leq \delta_{k_{i}-1} .
$$

According to Assumption 1 at the point $x^{*}$ for any $\varepsilon>0$ there exist $\beta>0$ and $\lambda_{0}>0$ such that

$$
D_{0}(y, \lambda) \subset \partial f\left(x^{*}+\bar{S}_{\varepsilon}\right)+S_{\varepsilon}
$$


for all $y \in S_{\beta}\left(x^{*}\right)$ and $\lambda \in\left(0, \lambda_{0}\right)$. Since the sequence $\left\{x^{k_{i}}\right\}$ converges to $x^{*}$ for $\beta>0$ there exists $i_{0}>0$ such that $x^{k_{i}} \in S_{\beta}\left(x^{*}\right)$ for all $i \geq i_{0}$. On the other hand since $\delta_{k}, \lambda_{k} \rightarrow 0$ as $k \rightarrow+\infty$ there exists $k_{0}>0$ such that $\delta_{k}<\varepsilon$ and $\lambda_{k}<\lambda_{0}$ for all $k>k_{0}$. Then there exists $i_{1} \geq i_{0}$ such that $k_{i} \geq k_{0}+1$ for all $i \geq i_{1}$. Thus it follows from (20) and (21) that

$$
\min _{v \in \partial f\left(x^{*}+\bar{S}_{\varepsilon}\right)}\|v\| \leq 2 \varepsilon
$$

Since $\varepsilon>0$ is arbitrary and the mapping $\partial f(x)$ is upper semicontinuous $0 \in \partial f\left(x^{*}\right)$.

Remark 7 Since Algorithm 1 can compute descent directions for any values of $\lambda>0$ we take $\lambda_{0} \in(0,1)$, some $\beta \in(0,1)$ and update $\lambda_{k}, k \geq 1$ as follows: $\lambda_{k}=\beta^{k} \lambda_{0}, k \geq 1$.

Remark 8 It follows from (16) and $c_{2} \leq c_{1}$ that always $\sigma_{s} \geq \lambda_{k}$ and therefore $\lambda_{k}>0$ is a lower bound for $\sigma_{s}$. This leads to the following rule for the computation of $\sigma_{s}$. We define a sequence:

$$
\theta_{m}=m \lambda_{k}, \quad m \geq 1
$$

and $\sigma_{s}$ is defined as the largest $\theta_{m}$ satisfying the inequality in Step 5 .

\section{Numerical experiments}

The efficiency of the proposed algorithm was verified by applying it to some academic test problems with nonsmooth objective functions. We consider three types of problems:

1. Problems with nonsmooth convex objective functions;

2. Problems with nonsmooth nonconvex regular objective functions;

3. Problems with nonsmooth, nonconvex and nonregular objective functions.

Test Problems 2.1-7, 2.9-12, 2.14-16, 2.18-21 and 2.23-25 from [20] and Problems $1-3,5$ and 7 from [4] have been used in numerical experiments. We also include the following problem with nonsmooth, nonconvex and nonregular objective function.

\section{Problem 1}

$$
\operatorname{minimize} f(x)=\sum_{i=1}^{20} \min _{j=1, \ldots, 5}\left\|x^{j}-a^{i}\right\|^{2}
$$

Here $x=\left(x^{1}, \ldots, x^{5}\right) \in \mathbb{R}^{15}$ and the vectors $a^{i} \in R^{3}, i=1, \ldots, 20$ are as follows:

\begin{tabular}{|c|c|c|c|c|c|c|c|c|c|}
\hline$a^{1}$ & $a^{2}$ & $a^{3}$ & $a^{4}$ & $a^{5}$ & $a^{6}$ & $a^{7}$ & $a^{8}$ & $a^{9}$ & $a^{10}$ \\
\hline 1.1 & 0.8 & 0.1 & 0.6 & -1.2 & 0.9 & 0.2 & -0.3 & -0.8 & 0.0 \\
\hline 1.0 & -1.6 & -1.0 & 0.2 & 1.0 & 1.9 & 0.2 & -0.2 & 0.6 & -0.4 \\
\hline-0.1 & 0.3 & -0.3 & 0.2 & 1.4 & -0.8 & 0.0 & 0.8 & -0.2 & 0.6 \\
\hline \hline$a^{11}$ & $a^{12}$ & $a^{13}$ & $a^{14}$ & $a^{15}$ & $a^{16}$ & $a^{17}$ & $a^{18}$ & $a^{19}$ & $a^{20}$ \\
\hline 1.0 & 0.0 & 0.0 & 2.1 & 0.2 & -2.1 & -1.0 & 0.3 & 1.1 & 3.1 \\
\hline 0.0 & 1.0 & 0.0 & -1.4 & -1.0 & 0.0 & 0.5 & -2.0 & 1.2 & -1.5 \\
\hline 0.0 & 0.0 & 1.0 & 1.0 & 1.0 & -1.0 & 1.5 & 0.9 & 1.0 & 2.1 \\
\hline
\end{tabular}


This function is well known clustering function (see $[8,9]$ ).

The brief description of these problems are given in Table 1, where the following notation is used:

$-n$ - number of variables;

- $n_{m}$ - the total number of functions under maximum and minimum (if the function contains maximum and minimum functions);

- $f_{\text {opt }}$ - optimum value (as reported in [20]).

Table 1 The description of problems

\begin{tabular}{lllll}
\hline Function type & Problems & $n$ & $n_{m}$ & $f_{\text {opt }}$ \\
\hline Nonsmooth & P1 (Problem 2.1 [20]) & 2 & 3 & 1.9522245 \\
convex & P2 (Problem 2.5 [20]) & 4 & 4 & -44 \\
& P3 (Problem 2.23 [20]) & 11 & 10 & 261.08258 \\
\hline & P4 (Problem 2.2 [20]) & 2 & 3 & 0 \\
& P5 (Problem 2.3 [20]) & 2 & 2 & 0 \\
& P6 (Problem 2.4 [20]) & 3 & 6 & 3.5997193 \\
& P7 (Problem 2.6 [20]) & 4 & 4 & -44 \\
& P8 (Problem 2.7 [20]) & 3 & 21 & 0.0042021 \\
& P9 (Problem 2.9 [20]) & 4 & 11 & 0.0080844 \\
Nonsmooth & P10 (Problem 2.10 [20]) & 4 & 20 & 115.70644 \\
nonconvex & P11 (Problem 2.11 [20]) & 4 & 21 & 0.0026360 \\
regular & P12 (Problem 2.12 [20]) & 4 & 21 & 0.0020161 \\
& P13 (Problem 2.14 [20]) & 5 & 21 & 0.0001224 \\
& P14 (Problem 2.15 [20]) & 5 & 30 & 0.0223405 \\
& P15 (Problem 2.16 [20]) & 6 & 51 & 0.0349049 \\
& P16 (Problem 2.18 [20]) & 9 & 41 & 0.0061853 \\
& P17 (Problem 2.19 [20]) & 7 & 5 & 680.63006 \\
& P18 (Problem 2.20 [20]) & 10 & 9 & 24.306209 \\
& P19 (Problem 2.21 [20]) & 20 & 18 & 133.72828 \\
& P20 (Problem 2.24 [20]) & 20 & 31 & 0.0000000 \\
& P21 (Problem 2.25 [20]) & 11 & 65 & 0.0480274 \\
\hline \multirow{4}{*}{ Nonsmooth } & P22 (Problem 1 [4]) & 2 & 6 & 2 \\
nonconvex & P23 (Problem 2 [4]) & 2 & - & 0 \\
nonregular & P24 (Problem 3 [4]) & 4 & - & 0 \\
& P25 (Problem 5 [4]) & 5 & - & 0 \\
& P26 (Problem 7 [4]) & 5 & - & 0 \\
& P27 (Problem 1) & 15 & 100 & 13.311214 \\
\hline & & & & \\
& & &
\end{tabular}

We compare the proposed algorithm with the subgradient method [26]. This method is as follows:

$$
x^{k+1}=x^{k}-\alpha_{k} v^{k}
$$

where $v^{k} \in \partial f\left(x^{k}\right)$ is any subgradient and $\alpha_{k}>0$ is a step-length.

Convergence of the subgradient method was proved only for convex functions [26]. However, we apply this algorithm also to nonconvex problems. We use two different versions of the subgradient method:

1. SUB1: in this version the step-length $\alpha_{k}$ is to some extent constant. We take $\alpha_{k}=$ 0.005 for the first 1000 iterations, $\alpha_{k}=0.001$ for the next 4000 iterations and $\alpha_{k}=0.0001$ for all other iterations. Such a choice of $\alpha_{k}$ leads to better results. 
2. SUB2: in this version the step-length $\alpha_{k}$ is a decreasing sequence. We take $\alpha_{k}=$ $1 / k$, however after each 25000 iterations we update it. Let $p_{k}$ is the largest integer, smaller than or equal to $k / 25000$. Then

$$
\alpha_{k}=\frac{1}{k-25000 p_{k}} .
$$

Without updating of $\alpha_{k}$ the convergence of the subgradient method is extremely poor for nonconvex functions.

Since there is no stopping criterion in the subgradient method we use the following two stopping criteria. The number of function evaluations is restricted by $10^{6}$ and also the algorithm stops if it cannot decrease the value objective function in 1000 successive iterations. We compute subgradients $v^{k}$ in (22) using the scheme from Section 3.

Numerical experiments were carried out on PC Pentium 4 with CPU $1.83 \mathrm{GHz}$ and $1 \mathrm{~GB}$ of RAM. We used 20 random starting points for each problem and starting points are the same for all three algorithms.

To compare the performance of the algorithms, we use two indicators: $n_{b}$ - the number of successful runs considering the best known solution and $n_{s}$ - the number of successful runs considering the best found solution by these three algorithms. For Problems P3 and P19 algorithms found better solutions than those reported in [20]. We take these new solutions as the best solutions. Assume that $f_{\text {opt }}$ and $\bar{f}$ are the values of the objective function at the best known solution and at the best found solution, respectively. Then we say that an algorithm finds the best solution with respect to a tolerance $\varepsilon>0$ if

$$
\frac{f_{*}-f_{0}}{1+\left|f_{*}\right|} \leq \varepsilon
$$

where $f_{*}$ is equal either to $f_{\text {opt }}$ (for $n_{b}$ ) or to $\bar{f}$ (for $n_{s}$ ) and $f_{0}$ is the optimal value of the objective function found by an algorithm. In our experiments $\varepsilon=10^{-4}$.

Results of numerical experiments are presented in Tables 2 and 3. In these tables ASM stands for the approximate subgradient method. In Table 2 we report the average objective function value over 20 runs of the algorithms as well as the numbers $n_{b}$ and $n_{s}$ for each problem. Table 3 presents the average number of the objective function evaluations and the average CPU time over 20 runs.

Results presented in Table 2 show that ASM outperforms other two algorithms in all problems except problems P5 and P7 where SUB2 algorithm produces better results. The latter with the application of updates of the step-lengths gives better results than SUB1 algorithm. Overall ASM produced best known solutions in $49.6 \%$ of cases $(100$ $\%$ for nonsmooth convex, $40.3 \%$ for nonconvex regular and $52.5 \%$ for nonconvex, nonregular functions). It gives in $81.9 \%$ of cases (100\% for nonsmooth convex, $73.3 \%$ for nonconvex regular and $98.3 \%$ for nonconvex, nonregular functions) best solutions among all three algorithms.

SUB1 algorithm produced best known solutions in $15.9 \%$ of cases (50\% for nonsmooth convex, $10.6 \%$ for nonconvex regular and $15 \%$ for nonconvex, nonregular functions). It gives in $19.1 \%$ of cases (50\% for nonsmooth convex, $15 \%$ for nonconvex regular and $15.8 \%$ for nonconvex, nonregular functions) best solutions among all three algorithms.

SUB2 algorithm produced best known solutions in $21.3 \%$ of cases ( $80 \%$ for nonsmooth convex, $11.9 \%$ for nonconvex regular and $20 \%$ for nonconvex, nonregular 
Table 2 Results of numerical experiments: obtained solutions

\begin{tabular}{lllllllllll}
\hline Prob. & \multicolumn{3}{c}{ ASM } & \multicolumn{3}{c}{ SUB1 } & \multicolumn{3}{c}{ SUB2 } \\
\cline { 2 - 9 } & $f_{a v}$ & $n_{b}$ & $n_{s}$ & $f_{a v}$ & $n_{b}$ & $n_{s}$ & $f_{a v}$ & $n_{b}$ & $n_{s}$ \\
\hline P1 & 1.95222 & 20 & 20 & 1.95236 & 18 & 18 & 1.95223 & 20 & 20 \\
P2 & -43.99997 & 20 & 20 & -43.94407 & 12 & 12 & -43.99973 & 20 & 20 \\
P3 & 3.70348 & 20 & 20 & 1106.25628 & 0 & 0 & 3.72973 & 8 & 8 \\
\hline P4 & 0.00492 & 18 & 19 & 2.72430 & 0 & 1 & 3.48299 & 6 & 6 \\
P5 & 0.26208 & 4 & 4 & 17.49696 & 14 & 17 & 0.08947 & 10 & 11 \\
P6 & 3.59972 & 20 & 20 & 3.60367 & 19 & 19 & 3.59974 & 20 & 20 \\
P7 & 12.17732 & 11 & 12 & -11.53027 & 5 & 5 & -29.89826 & 7 & 12 \\
P8 & 0.03891 & 3 & 13 & 0.04810 & 0 & 3 & 0.04761 & 0 & 8 \\
P9 & 0.04273 & 0 & 17 & 1.59493 & 0 & 1 & 0.09259 & 0 & 3 \\
P10 & 115.70646 & 20 & 20 & 231.41148 & 0 & 0 & 197.90843 & 0 & 0 \\
P11 & 0.00318 & 0 & 20 & 0.79443 & 0 & 0 & 0.21997 & 0 & 0 \\
P12 & 0.06862 & 0 & 12 & 0.10392 & 0 & 3 & 0.06876 & 0 & 8 \\
P13 & 0.10373 & 2 & 19 & 4.85194 & 0 & 0 & 1.75332 & 0 & 1 \\
P14 & 0.33172 & 2 & 17 & 12.30531 & 0 & 1 & 5.94889 & 0 & 2 \\
P15 & 0.04008 & 17 & 20 & 0.59012 & 0 & 0 & 0.50676 & 0 & 0 \\
P16 & 0.14117 & 0 & 20 & 0.52659 & 0 & 0 & 0.47843 & 0 & 0 \\
P17 & 680.63056 & 20 & 20 & 1283.63922 & 0 & 0 & 738.18848 & 0 & 0 \\
P18 & 24.30702 & 20 & 20 & 1083.73783 & 0 & 0 & 242.21861 & 0 & 0 \\
P19 & 93.93033 & 8 & 20 & 6353.92360 & 0 & 0 & 3597.49593 & 0 & 0 \\
P20 & 0.38112 & 0 & 20 & 749.33916 & 0 & 0 & 172.41626 & 0 & 0 \\
P21 & 0.34827 & 0 & 14 & 1.52721 & 0 & 4 & 0.95114 & 0 & 6 \\
\hline P22 & 2.00000 & 20 & 20 & 2.00009 & 18 & 18 & 2.00000 & 20 & 20 \\
P23 & 0.40000 & 12 & 19 & 2.12330 & 0 & 1 & 2.13230 & 0 & 1 \\
P24 & 0.90000 & 11 & 19 & 54.55650 & 0 & 0 & 44.29527 & 0 & 1 \\
P25 & 0.00000 & 20 & 20 & 7.09383 & 0 & 0 & 1.11665 & 4 & 4 \\
P26 & 0.31303 & 0 & 20 & 62.26375 & 0 & 0 & 37.87321 & 0 & 0 \\
P27 & 26.19072 & 0 & 20 & 42.35418 & 0 & 0 & 40.92745 & 0 & 0 \\
\hline & & & & & & & & 0
\end{tabular}

functions). It gives in $28.0 \%$ of cases ( $80 \%$ for nonsmooth convex, $21.4 \%$ for nonconvex regular and $21.7 \%$ for nonconvex, nonregular functions) best solutions among all three algorithms.

Comparing these results one can see that the approximate subgradient algorithm is more efficient than two other subgradient algorithms. Our results show that both versions of the subgradient method are inefficient for solving nonsmooth optimization problems with moderately large number of variables (more than 10 variables).

One can see from results presented in Table 3 that ASM requires significantly less number of the objective function evaluations. However this is not the case for average CPU time. In Problems P2, P4, P7, P17, P18, P19, P24, P25 it requires more CPU time than other two algorithms. This means that in this problems ASM spends the most of CPU time to solve the subproblem to find descent directions. In the same time in the most of these problems it produces significantly better results than other algorithms.

\section{Conclusions}

In this paper we proposed an approximate subgradient algorithm for solving unconstrained nonsmooth convex and nonconvex optimization problems. The problem of 
Table 3 Results of numerical experiments: the number of function evaluations

\begin{tabular}{lllllll}
\hline \multirow{2}{*}{ Prob. } & \multicolumn{3}{c}{ No. of function eval. } & \multicolumn{3}{c}{ CPU time } \\
\cline { 2 - 7 } & ASM & SUB1 & SUB2 & ASM & SUB1 & SUB2 \\
\hline P1 & 365 & 180761 & 69601 & 0.03 & 0.03 & 0.01 \\
P2 & 1600 & 954382 & 369713 & 0.17 & 0.12 & 0.04 \\
P3 & 5419 & 1000000 & 878419 & 1.38 & 16.59 & 14.55 \\
\hline P4 & 4165 & 241993 & 31460 & 0.38 & 0.03 & 0.01 \\
P5 & 780 & 606317 & 577381 & 0.04 & 0.11 & 0.11 \\
P6 & 643 & 756612 & 283865 & 0.08 & 0.09 & 0.03 \\
P7 & 2875 & 819993 & 643470 & 0.24 & 0.11 & 0.07 \\
P8 & 692 & 992131 & 582893 & 0.05 & 2.94 & 1.72 \\
P9 & 1691 & 905838 & 880996 & 0.13 & 0.29 & 0.27 \\
P10 & 1736 & 1000000 & 1000000 & 0.23 & 3.14 & 3.11 \\
P11 & 3815 & 957729 & 934791 & 0.36 & 0.82 & 0.80 \\
P12 & 2324 & 751279 & 638933 & 0.22 & 2.78 & 2.36 \\
P13 & 2788 & 996645 & 930583 & 0.42 & 2.13 & 1.95 \\
P14 & 3231 & 1000000 & 870773 & 0.46 & 4.51 & 3.91 \\
P15 & 5662 & 977514 & 946413 & 0.89 & 11.15 & 10.69 \\
P16 & 11197 & 889630 & 725645 & 1.93 & 12.78 & 10.41 \\
P17 & 2930 & 1000000 & 1000000 & 0.56 & 0.20 & 0.15 \\
P18 & 11454 & 1000000 & 1000000 & 2.47 & 0.29 & 0.21 \\
P19 & 34949 & 1000000 & 1000000 & 18.63 & 0.70 & 0.34 \\
P20 & 50617 & 1000000 & 1000000 & 28.16 & 61.23 & 55.77 \\
P21 & 6053 & 620464 & 558975 & 1.38 & 15.33 & 13.59 \\
\hline P22 & 339 & 82720 & 41532 & 0.03 & 0.02 & 0.01 \\
P23 & 378 & 955895 & 527285 & 0.04 & 0.07 & 0.04 \\
P24 & 1487 & 967631 & 885073 & 0.22 & 0.07 & 0.06 \\
P25 & 7989 & 1000000 & 960136 & 1.71 & 0.17 & 0.15 \\
P26 & 11934 & 1000000 & 1000000 & 2.56 & 3.75 & 3.96 \\
P27 & 6671 & 54272 & 33675 & 1.77 & 1.26 & 1.59 \\
\hline & & & & & &
\end{tabular}

computation of descent directions in this algorithm is reduced to the minimization of a convex piecewise linear function. The latter problem is solved using the subgradient method. Unlike the subgradient method the proposed algorithm may use more than one approximate subgradient at each iteration. This makes it similar to bundle-type algorithms. But on the same time it does not use polyhedral underestimators of the objective function which makes it different from them. Moreover, in this method the subgradient method is applied to find descent directions. This makes the proposed method easier to implement. However, we cannot say that this algorithm is as efficient as bundle-type algorithms. To make the proposed algorithm more efficient better algorithms for solving subproblems should be developed. This as well as the comparison of the proposed algorithm with the bundle method will be a topic for the future research.

Acknowledgements Dr. Adil Bagirov is the recipient of an Australian Research Council Australian Research Fellowship (Project number: DP 0666061).

\section{References}

1. Bagirov A.M. and Gasanov A.A. (1995) A method of approximating a quasidifferential, Journal of Computational Mathematics and Mathematical Physics, 35(4): 403-409. 
2. Bagirov A.M. (1998) Discrete gradient as applied to the minimization of Lipschitzian functions, Journal of Computational Mathematics and Mathematical Physics, 38(10): 15561565.

3. Bagirov A.M. (1999) Minimization methods for one class of nonsmooth functions and calculation of semi-equilibrium prices, In: A. Eberhard et al. (eds.) Progress in Optimization: Contribution from Australasia, Kluwer Academic Publishers, 1999, 147-175.

4. Bagirov A.M. (2002) A method for minimizing of quasidifferentiable functions, Optimization Methods and Software, 17(1): 31-60.

5. Bagirov A.M. (2003) Continuous subdifferential approximations and their applications, Journal of Mathematical Sciences, 115(5): pp. 2567-2609.

6. Bagirov A.M., Ghosh, M and Webb, D (2006) A derivative-free method for linearly constrained nonsmooth optimization, Journal of Industrial and Management Optimization, 2(3): pp. 319-338.

7. Bagirov A.M., Karasozen B and Sezer M (2007) Discrete gradient method: a derivative free method for nonsmooth optimization, Journal of Optimization Theory and Applications, accepted for publication.

8. Bagirov A.M., Rubinov A.M., Soukhoroukova N.V. and Yearwood J (2003) Supervised and unsupervised data classification via nonsmooth and global optimisation, TOP: Spanish Operations Research Journal, 11(1): pp. 1-93.

9. Bagirov A.M. and Yearwood J (2006) A new nonsmooth optimization algorithm for minimum sum-of-squares clustering problems, European Journal of Operational Research, 170(2): pp. 578-596.

10. Bertsekas D.P. (1999) Nonlinear Programming, Athena Scientific, 2-nd edition, 1999.

11. Burke J.V., Lewis A.S. and Overton M.L. (2005) A robust gradient sampling algorithm for nonsmooth, nonconvex optimization, SIAM Journal on Optimization, 15(3): pp. 751-779.

12. Clarke F.H. (1983) Optimization and Nonsmooth Analysis, New York, John Wiley.

13. Demyanov V.F. and Rubinov A.M. (1995) Constructive Nonsmooth Analysis, Peter Lang, Frankfurt am Main.

14. Frangioni A (2002) Generalized bundle methods, SIAM Journal on Optimization, 113(1): pp. 117-156.

15. Golikov A.I. and Evtushenko, Yu.G. (2001) A new method for solving systems of linear equalities and inequalities, Doklady Mathematics, 64(3): pp. 370-373.

16. Gaudioso M and Monaco M.F. (1982) A bundle type approach to the unconstrained minimization of convex nonsmooth functions, Mathematical Programming, 23: pp. 216-226.

17. Hiebert K (1980) Solving systems of linear equations ans inequalities, SIAM Journal on Numerical Analysis, 17(3): pp. 447-464.

18. Hiriart-Urruty J.B. and Lemarechal C. (1993) Convex Analysis and Minimization Algorithms, Springer Verlag, New York, Volumes 1, 2.

19. Kiwiel K.C. (1985) Methods of Descent for Nondifferentiable Optimization, Lecture Notes in Mathematics, Springer-Verlag, Berlin, Volume 1133.

20. Luksan, L. and Vlcek, J., Test Problems for Nonsmooth Unconstrained and Linearly Constrained Optimization, Technical Report No. 78, Institute of Computer Science, Academy of Sciences of the Czech Republic, 2000.

21. Makela M.M. and Neittaanmaki P. (1992) Nonsmooth Optimization, World Scientific, Singapore.

22. Mifflin R (1977) Semismooth and semiconvex functions in constrained optimization, SIAM J. Control and Optimization, 15(6): pp. 959-972.

23. Mifflin R (1977) An algorithm for constrained optimization with semismooth functions, Mathematics of Operations Research, 2: pp. 191-207.

24. Polak E and Royset J.O. (2003) Algorithms for finite and semi-infinite min-max-min problems using adaptive smoothing techniques, Journal of Optimization Theory and Applications, 119(3): pp. 421-457.

25. Polyak B (1985) Introduction to Optimization, Optimization Software, New York.

26. Shor N.Z. (1985) Minimization methods for non-differentiable functions, Berlin, New York, Springer-Verlag.

27. Wolfe P.H. (1975) A method of conjugate subgradients of minimizing nondifferentiable convex functions, Mathematical Programming Study, 3: pp. 145-173.

28. Zowe J (1985) Nondifferentiable optimization: A motivation and a short introduction into the subgradient and the bundle concept, In: NATO SAI Series, Vol. 15, Computational Mathematical Programming, Schittkowski, K., (ed.), pp. 323-356, Springer-Verlag, New York. 